\title{
Numerical Analysis of DAEs from Coupled Circuit and Semiconductor Simulation *
}

\author{
Monica Selva Soto ${ }^{\mathrm{a}}$ and Caren Tischendorf ${ }^{\mathrm{a}}$ \\ ${ }^{a}$ Humboldt University of Berlin, Institut for Mathematics
}

\begin{abstract}
In this work we are interested in the numerical solution of a coupled model of differential algebraic equations (DAEs) and partial differential equations (PDEs). The DAEs describe the behavior of an electrical circuit that contains semiconductor devices and the partial differential equations constitute drift-diffusion equations modeling the semiconductor devices in the circuit.

After space discretization using a finite element method, the coupled system results in a differential-algebraic system with a properly stated leading term. We investigate the structure and the properties of this DAE system. In particular, we develop structural criteria for the DAE index. This is of basic interest since DAE properties like stability, existence and uniqueness of solutions depend strongly on its index.
\end{abstract}

Key words: differential algebraic equation, partial differential equation, tractability index, modified nodal analysis, drift-diffusion equations

\section{Introduction}

Nowadays semiconductor devices in an electrical circuit are modeled by small circuits containing basic network elements (capacitors, resistors, inductors, voltage and current sources) described by algebraic and ordinary differential equations. But these equivalent circuits may depend on hundreds of parameters and its correct adjustment has become a very difficult task for the network design. This has motivated the idea of using distributed device models, represented by a system of partial differential equations, to describe the behavior

\footnotetext{
ऋ Supported by the DFG Research Center "Mathematics for key technologies" (FZT86) in Berlin.

Email addresses: monica@mathematik.hu-berlin.de (Monica Selva Soto), caren@mathematik.hu-berlin.de (Caren Tischendorf).
} 
of the semiconductor devices within the circuit [1]. The resulting mathematical models couple the differential algebraic equations (DAEs) describing the behavior of circuit and the partial differential equations (PDEs) modeling semiconductor devices.

In this work we are interested in the numerical solution of the system that is obtained when high frequency devices in an electrical circuit are modeled via drift-diffusion equations. In section 2 the equations resulting from the Modified Nodal Analysis (MNA) of the circuit are explained. The drift-diffusion equations are presented in section 3 as well as its discretization by a finite element method.

Finally, in section 4 the DAE that results from the coupling of the MNA equations and the discrete drift-diffusion equations is constructed and its index is studied. The knowledge about the DAE index allows us to determine the conditions that consistent initial values must satisfy and which numerical methods are feasible for its solution.

\section{Circuit Equations}

The mathematical model that results from modified nodal analysis applied to an electrical network containing resistors, capacitors, inductors and independent voltage and current sources ${ }^{1}$ has the form [3]

$$
\begin{aligned}
A_{C} \frac{\mathrm{d}}{\mathrm{d} t} q_{C}\left(A_{C}^{T} e, t\right)+A_{R} g\left(A_{R}^{T} e, t\right)+A_{L} j_{L}+A_{V} j_{V}+A_{I} i_{S}(t) & =0, \\
\frac{\mathrm{d}}{\mathrm{d} t} \phi\left(j_{L}, t\right)-A_{L}^{T} e & =0, \\
A_{V}^{T} e-v_{S}(t) & =0 .
\end{aligned}
$$

The unknowns $e(t): \mathbb{R} \rightarrow \mathbb{R}^{n_{N}}, j_{L}(t): \mathbb{R} \rightarrow \mathbb{R}^{n_{L}}$ and $j_{V}(t): \mathbb{R} \rightarrow \mathbb{R}^{n_{V}}$ represent the node potentials, excepting the mass node, the currents through inductors and the currents through voltage sources respectively. The matrices $A_{C}, A_{R}, A_{L}, A_{V}$ and $A_{I}$ are the element-related (reduced) incidence matrices, they have entries from $\{-1,0,1\}$. Let the following assumptions on the circuit equations be satisfied in the forthcoming sections:

(1) the input functions $v_{S}(t)$ and $i_{S}(t)$, associated to the independent voltage and current sources respectively, are continuous,

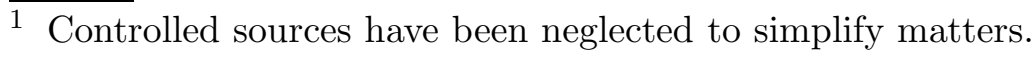


(2) the functions $q_{C}(u, t), \phi(j, t)$ and $g(u, t)$ are continuously differentiable and have positive definite partial Jacobians

$$
C(u, t)=\frac{\partial q_{C}(u, t)}{\partial u}, \quad L(j, t)=\frac{\partial \phi(j, t)}{\partial j}, \quad G(u, t)=\frac{\partial g(u, t)}{\partial u},
$$

(3) and the circuit contains neither loops of voltage sources only nor cut sets of current sources only. These two conditions hold if and only if the matrices $A_{V}$ and $\left(A_{C} A_{R} A_{L} A_{V}\right)^{T}$ have full column rank, respectively.

The second assumption concerning the Jacobians reflects local passivity of capacitances, inductances and resistances [4]. The third assumption is necessary from the electric point of view in order to prevent short-circuits.

Under these assumptions it was shown $[17,3]$ that the index of the circuit equations (1)-(3) does not exceed two. More precisely, the index equals two if and only if the circuit contains LI-cut sets (cut sets of inductors and current sources) or CV-loops (loops of capacitors and voltage sources) with at least one voltage source.

Additionally, the previous assumptions allow the circuit equation systems to be formulated as DAEs with a properly stated leading term [14].

\section{Drift-Diffusion Equations}

We will consider the non-stationary drift-diffusion model of a semiconductor device. For convenience, we formulate the model equations in only one spatial dimension. The segment $\bar{\Omega}=[0, l] \subset \mathbb{R}$ describes the range of the device, including its contacts and $t \in\left[t_{a}, t_{b}\right]$ represents the time. The model equations are given by the Poisson equation

$$
-\frac{\partial}{\partial x}\left(\varepsilon \frac{\partial \psi}{\partial x}\right)=q(C+p-n), \quad \forall x \in \Omega
$$

for the electrostatic potential $\psi=\psi(x, t)$ and the continuity equations

$$
-q \frac{\partial n}{\partial t}+\frac{\partial J_{n}}{\partial x}=q R, \quad q \frac{\partial p}{\partial t}+\frac{\partial J_{p}}{\partial x}=-q R, \quad \forall x \in \Omega
$$

for the densities of electrons $n=n(x, t)$ and holes $p=p(x, t)$. In (4a)-(4b) $\varepsilon$ represents the dielectric constant and $q$ is the elementary charge. The current densities caused by electrons and holes, $J_{n}$ and $J_{p}$ respectively, can be described as a composition of a drift and a diffusion current,

$$
J_{n}=q \mu_{n}\left(U_{T} \frac{\partial n}{\partial x}-n \frac{\partial \psi}{\partial x}\right), \quad J_{p}=-q \mu_{p}\left(U_{T} \frac{\partial p}{\partial x}+p \frac{\partial \psi}{\partial x}\right) .
$$


In the previous expressions $U_{T}$ is the thermal voltage, it depends on the Boltzmann constant, the elementary charge and the temperature $T$ of the semiconductor. Here we will consider $T$ as a constant. The electrons and holes mobilities, $\mu_{n}$ and $\mu_{p}$ respectively, are assumed to be nonnegative, bounded functions of $x$.

In (4a) $C=C(x)$ is the doping profile of the semiconductor. The function $R$ in (4b) describes the balance of generation and recombination of electrons and holes. Frequently used models for $R$ are the Shockley-Read-Hall recombination $R_{S H R}$ and the Auger recombination $R_{A u}$,

$$
\begin{aligned}
& R_{S R H}=\frac{n p-\eta_{i}^{2}}{\tau_{n}\left(p+\eta_{i}\right)+\tau_{p}\left(n+\eta_{i}\right)}, \quad R_{A u}=\left(n p-\eta_{i}^{2}\right)\left(C_{n} n+C_{p} p\right), \\
& R=R_{S H R}+R_{A u},
\end{aligned}
$$

where $\tau_{n}$ and $\tau_{p}$ reflect the average lifetimes of electrons and holes. The socalled intrinsic concentration $\eta_{i}$ is the geometric average of the carrier concentrations in a semiconductor in equilibrium and $C_{n}$ and $C_{p}$ are the Auger coefficients.

For a more detailed description of mathematical models for semiconductors and results about existence and uniqueness of solutions of these models see e.g. $[15,16,5,12,6,8]$.

The electric currents

$$
\left(\begin{array}{c}
j_{0}(t) \\
j_{l}(t)
\end{array}\right)=\left(\begin{array}{c}
J(0, t) \\
-J(l, t)
\end{array}\right) \text { with } J(x, t)=J_{n}(x, t)+J_{p}(x, t)-\varepsilon \frac{\partial}{\partial t} \frac{\partial \psi}{\partial x}(x, t),
$$

represent the semiconductor's output to the potentials applied to its boundary. The values of $j_{0}(t)$ and $j_{l}(t)$ satisfy $j_{l}(t)=-j_{0}(t), \forall t \in\left[t_{a}, t_{b}\right]^{2}$. This means that we may choose one of the terminals of the semiconductor device as reference terminal, let us say the terminal at $x=l$, the current through it may be calculated in terms of the current leaving the other terminal. In what follows we will refer to $j_{0}(t)$ as the semiconductor's current.

Because the dependent variables in (4) are of highly different orders of magnitude and show a strongly different behavior in regions with small and large space charge, two scalings are recommended in [16]. The scaled one-dimensional

$\overline{2}$ This is a consequence of charge conservation. Differentiating (4a) with respect to time and adding $(4 \mathrm{~b})$ yields $\frac{\partial J}{\partial x} \equiv 0$. 
Poisson equation ${ }^{3}$ reads

$$
-\frac{\partial}{\partial x}\left(\lambda^{2} \frac{\partial \psi}{\partial x}\right)=C-n+p, \quad \lambda^{2}=\frac{\varepsilon U_{T}}{q C_{0} x_{s}^{2}} .
$$

The constants $C_{0}$ and $x_{s}$ depend on the scaling. The continuity equations change to

$$
\begin{array}{ll}
-\frac{\partial n}{\partial t}+\frac{\partial J_{n}}{\partial x}=R, & J_{n}=\mu_{n}\left(\frac{\partial n}{\partial x}-n \frac{\partial \psi}{\partial x}\right) \\
\frac{\partial p}{\partial t}+\frac{\partial J_{p}}{\partial x}=-R, & J_{p}=-\mu_{p}\left(\frac{\partial p}{\partial x}+p \frac{\partial \psi}{\partial x}\right)
\end{array}
$$

and the boundary and initial conditions for the scaled model are

$$
\begin{array}{cl}
\psi(0, t)=\frac{1}{U_{T}}\left(\psi_{b i}(0)+\omega_{0}(e(t)),\right. & \psi(l, t)=\frac{1}{U_{T}}\left(\psi_{b i}(l)+\omega_{l}(e(t))\right. \\
n(0, t)=\frac{C(0)+\sqrt{C(0)^{2}+4 \eta_{i}^{2}}}{2}, & n(l, t)=\frac{C(l)+\sqrt{C(l)^{2}+4 \eta_{i}^{2}}}{2} \\
p(0, t)=\frac{-C(0)+\sqrt{C(0)^{2}+4 \eta_{i}^{2}}}{2}, & p(l, t)=\frac{-C(l)+\sqrt{C(l)^{2}+4 \eta_{i}^{2}}}{2} \\
n\left(x, t_{a}\right)=n_{a}(x), & p\left(x, t_{a}\right)=p_{a}(x) .
\end{array}
$$

The function $\psi_{b i}(x)$ is the built-in potential and $\omega_{0}, \omega_{l}$ are the externally applied biases. In this work we want to consider the semiconductor devices as part of an electrical circuit modeled by (1)-(3). Then the biases applied to the semiconductor boundaries depend on the node potentials of the circuit, that is why in (5d) we have written $\omega_{0}$ and $\omega_{l}$ as functions of $e$.

The current of the semiconductor in terms of the scaled variables is

$$
j_{0}(t)=\frac{q \mu_{0} U_{T} C_{0}}{x_{s}} J(0, t)=\frac{q \mu_{0} U_{T} C_{0}}{x_{s}}\left(J_{n}(0, t)+J_{p}(0, t)-\lambda^{2} \frac{\partial}{\partial x} \frac{\partial \psi}{\partial t}(0, t)\right),
$$

where $\mu_{0}$ is a constant that also depends on the scaling.

In [7] it is pointed out that not only for the numerical solution of this problem, but also for the study of its analytical properties, it is convenient to replace the Poisson equation (5a) by the energy conservation equation

$$
\frac{\partial J}{\partial x}=\frac{\partial}{\partial x}\left(J_{n}+J_{p}-\lambda^{2} \frac{\partial}{\partial x} \frac{\partial \psi}{\partial t}\right)=0
$$

$\overline{3}$ The scaled variables and constants have been named as the original ones. 
that is obtained after differentiation of the Poisson equation with respect to time and elimination of $\frac{\partial n}{\partial t}$ and $\frac{\partial p}{\partial t}$ from the continuity equations. If the initial value for $\psi\left(x, t_{a}\right)=\psi_{a}(x)$ is chosen such that the functions $\psi_{a}(x), n_{a}(x)$ and $p_{a}(x)$ satisfy the Poisson equation,

$$
-\frac{\partial}{\partial x}\left(\lambda^{2} \frac{\partial \psi_{a}}{\partial x}\right)=q\left(C+p_{a}-n_{a}\right)
$$

the equivalence between (5a) and (6) is guaranteed [7].

\subsection{Finite Element Method for the Numerical Solution of the Drift-Diffusion Equations}

The functions $(\psi(x, t), n(x, t), p(x, t))$ are a weak solution of $(5)$ if

$$
\psi(x, t), n(x, t), p(x, t) \in L_{2}\left(\left(t_{a}, t_{b}\right), H^{1}(\Omega)\right),
$$

have generalized derivatives $\frac{\partial n}{\partial t}, \frac{\partial p}{\partial t} \in L_{2}\left(\left(t_{a}, t_{b}\right), L_{2}(\Omega)\right)$ and satisfy the equations

$$
\begin{gathered}
\lambda^{2} \int_{0}^{l} \frac{\partial \psi}{\partial x} \frac{\partial \varphi}{\partial x} \mathrm{~d} x=\int_{0}^{l}(C-n+p) \varphi \mathrm{d} x, \\
-\int_{0}^{l} \frac{\partial n}{\partial t} \varphi \mathrm{d} x-\int_{0}^{l} J_{n} \frac{\partial \varphi}{\partial x} \mathrm{~d} x=\int_{0}^{l} R \varphi \mathrm{d} x, \\
\int_{0}^{l} \frac{\partial p}{\partial t} \varphi \mathrm{d} x-\int_{0}^{l} J_{p} \frac{\partial \varphi}{\partial x} \mathrm{~d} x=-\int_{0}^{l} R \varphi \mathrm{d} x,
\end{gathered}
$$

for all functions $\varphi \in H_{0}^{1}(\Omega)$ and almost all $t \in\left[t_{a}, t_{b}\right]$ as well as the boundary and initial conditions in $(5 \mathrm{~d})-(5 \mathrm{~g})$.

An approximation $\left(\psi_{h}(x, t), n_{h}(x, t), p_{h}(x, t)\right)$ of the weak solution of this problem can be determined by the finite element method. For sake of simplicity, let us divide the interval $[0, l]$ into equally-spaced subintervals $\left[x_{i-1}, x_{i}\right]$ with $h=x_{i}-x_{i-1}, x_{1}=0, x_{m}=l$ for $i=2, \ldots, m$. Using the Galerkin ansatz

$$
\psi_{h}(x, t)=\sum_{j=1}^{m} \psi_{j}(t) \varphi_{j}(x)
$$

the function $\psi_{h}(x, t)$ is obtained by solving the system consisting of the equation (8a) for all basis functions $\varphi_{i}(x), i=2, \ldots, m-1$ as well as the boundary and initial conditions in $(5 \mathrm{~d})-(5 \mathrm{~g})$. As basis functions $\varphi_{i}(x)$ we choose the 
polynomials of degree one satisfying

$$
\varphi_{i}\left(x_{j}\right)= \begin{cases}1, & \text { if } i=j \\ 0, & \text { else }\end{cases}
$$

for all $j=1,2, \ldots, m$. The integral in the right-hand-side of (8a) is approximated with the trapezoidal rule.

\subsubsection{Discretization of the Continuity Equations}

To obtain the approximations $n_{h}(x, t)$ and $p_{h}(x, t)$ equations $(8 \mathrm{~b})$ and $(8 \mathrm{c})$ are not discretized in the usual way, but by the so-called Scharfetter-Gummel discretization [15]. This way, the area of convergence is usually larger than that one for the standard discretization. The Scharfetter-Gummel discretization is based on the assumption that $J_{n}(x, t)$ and $J_{p}(x, t)$ can be approximated by constant functions on each subinterval $\left(x_{j-1}, x_{j}\right], j=2, \ldots, m$.

Let us denote by $J_{n, h}(x, t), J_{p, h}(x, t), \mu_{n, h}$ and $\mu_{p, h}$ the piecewise constant functions that approximate $J_{n}(x, t), J_{p}(x, t), \mu_{n}$ and $\mu_{p}$, respectively,

$$
\begin{aligned}
J_{n, h}(x, t) & =J_{n}^{j}(t), \quad J_{p, h}(x, t)=J_{p}^{j}(t), \\
\mu_{n, h}(x) & =\mu_{n}^{j}=\mu_{n}\left(x_{j-1}+h / 2\right), \\
\mu_{p, h}(x) & =\mu_{p}^{j}=\mu_{p}\left(x_{j-1}+h / 2\right)
\end{aligned}
$$

for $x \in\left(x_{j-1}, x_{j}\right]$. The relation

$$
\mu_{n}^{j}\left(\frac{\partial n_{h}}{\partial x}-n_{h} \frac{\partial \psi_{h}}{\partial x}\right)=J_{n}^{j}
$$

with $x \in\left[x_{j-1}, x_{j}\right]$ and initial value $n_{h}\left(x_{j-1}, t\right)=n_{j-1}$ is an initial value problem (IVP) that can be solved for $n_{h}(x, t)$. Its solution is

$$
n_{h}(x, t)= \begin{cases}e^{\left(\psi_{j}-\psi_{j-1}\right)\left(x-x_{j-1}\right) / h}\left(n_{j-1}+\frac{J_{n}^{j}}{\mu_{n}^{j}}\left(\frac{h}{\psi_{j}-\psi_{j-1}}\right)\right) & -\frac{J_{n}^{j}}{\mu_{n}^{j}}\left(\frac{h}{\psi_{j}-\psi_{j-1}}\right) \\ n_{j-1}+\frac{J_{n}^{j}}{\mu_{n}^{j}}\left(x-x_{j-1}\right) & \text { if } \psi_{j} \neq \psi_{j-1}\end{cases}
$$

Evaluating $n_{h}(x, t)$ at $x=x_{j}$, an expression for $J_{n}^{j}$ in terms of $\psi_{j-1}, \psi_{j}, n_{j-1}$ and $n_{j}$ is obtained. In a similar way, $J_{p}^{j}$ can be calculated in terms of $\psi_{j-1}$, $\psi_{j}, p_{j-1}$ and $p_{j}$.

Replacing $\varphi$ by the basis functions $\varphi_{j}$ in (8b)-(8c) yields 


$$
\begin{gathered}
-\int_{x_{j-1}}^{x_{j+1}} \frac{\partial n}{\partial t} \varphi_{j} \mathrm{~d} x-J_{n}^{j} \int_{x_{j-1}}^{x_{j}} \frac{\partial \varphi_{j}}{\partial x} \mathrm{~d} x-J_{n}^{j+1} \int_{x_{j}}^{x_{j+1}} \frac{\partial \varphi_{j}}{\partial x} \mathrm{~d} x=\int_{x_{j-1}}^{x_{j+1}} R \varphi_{j} \mathrm{~d} x \\
\int_{x_{j-1}}^{x_{j+1}} \frac{\partial p}{\partial t} \varphi_{j} \mathrm{~d} x-J_{p}^{j} \int_{x_{j-1}}^{x_{j}} \frac{\partial \varphi_{j}}{\partial x} \mathrm{~d} x-J_{p}^{j+1} \int_{x_{j}}^{x_{j+1}} \frac{\partial \varphi_{j}}{\partial x} \mathrm{~d} x=-\int_{x_{j-1}}^{x_{j+1}} R \varphi_{j} \mathrm{~d} x
\end{gathered}
$$

for $j=2,3, \ldots, m-1$. Inserting the expressions obtained above for $J_{n}^{j}$ and $J_{p}^{j}$ into the last equations and approximating the integrals that contain partial derivatives with respect to time and those in the right-hand-sides by the trapezoidal rule we obtain an IVP for the coefficients that define $n_{h}(x, t)$ and $p_{h}(x, t)$

$$
\begin{gathered}
-h \frac{\mathrm{d}}{\mathrm{d} t} n_{j}+\frac{1}{h} \mu_{n}^{j+1} f\left(z_{j+1}\right) n_{j+1}-\frac{1}{h}\left(\mu_{n}^{j+1} f\left(-z_{j+1}\right)+\mu_{n}^{j} f\left(z_{j}\right)\right) n_{j} \\
+\frac{1}{h} \mu_{n}^{j} f\left(-z_{j}\right) n_{j-1}-h R_{j}=0 \\
h \frac{\mathrm{d}}{\mathrm{d} t} p_{j}-\frac{1}{h} \mu_{p}^{j+1} f\left(-z_{j+1}\right) p_{j+1}+\frac{1}{h}\left(\mu_{p}^{j+1} f\left(z_{j+1}\right)+\mu_{p}^{j} f\left(-z_{j}\right)\right) p_{j} \\
-\frac{1}{h} \mu_{p}^{j} f\left(z_{j}\right) p_{j-1}+h R_{j}=0, \\
n_{1}=n(0, t), n_{m}=n(l, t), p_{1}=p(0, t), p_{m}=p(l, t), \\
n_{j}\left(t_{a}\right)=n_{a}\left(x_{j}\right), p_{j}\left(t_{a}\right)=p_{a}\left(x_{j}\right), \quad \text { for } j=2,3, \ldots, m-1 .
\end{gathered}
$$

In the first two equations, $z_{j}=\psi_{j}-\psi_{j-1}$ and

$$
f(z)= \begin{cases}\frac{z}{e^{z}-1}, & \text { if } z \neq 0 \\ 1, & \text { else }\end{cases}
$$

The function $n_{h}(x, t)$ has the form, for $x \in\left(x_{j-1}, x_{j}\right]$ and $t \in\left[t_{a}, t_{b}\right]$,

$$
n_{h}(x, t)= \begin{cases}n_{j}\left(\frac{e^{z_{j}\left(x-x_{j-1}\right) / h}-1}{e^{z_{j}}-1}\right)-n_{j-1}\left(\frac{e^{z_{j}\left(x-x_{j-1}\right) / h}-e^{z_{j}}}{e^{z_{j}}-1}\right), & \text { if } z_{j} \neq 0 \\ n_{j-1}+\left(n_{j}-n_{j-1}\right) \frac{x-x_{j-1}}{h}, & \text { else. }\end{cases}
$$

The current of the semiconductor can be approximated by

$$
j_{0}(t) \approx \frac{q \mu_{0} U_{T} C_{0}}{x_{s}}\left(J_{n, h}(0, t)+J_{p, h}(0, t)-\lambda^{2} \frac{\partial}{\partial t} \frac{\partial \psi_{h}(0, t)}{\partial x}\right)=j_{S}^{c}-\frac{\mathrm{d}}{\mathrm{d} t} j_{S}^{d},
$$

where $j_{S}^{c}$ denotes the conduction current and the derivative of $j_{S}^{d}$ with respect to the time, the displacement current, 


$$
\begin{aligned}
& j_{S}^{c}=\alpha\left(J_{n, h}(0, t)+J_{p, h}(0, t)\right), \quad \alpha=\frac{q \mu_{0} U_{T} C_{0}}{x_{s}} \\
& j_{S}^{d}=\beta\left(\psi_{2}(t)-\psi_{1}(t)\right)=\beta\left(\psi_{2}(t)-\frac{1}{U_{T}}\left(\psi_{b i}(0)+\omega_{0}(e, t)\right)\right), \quad \beta=\alpha \frac{\lambda^{2}}{h} .
\end{aligned}
$$

If standard finite elements are used to obtain the approximations $n_{h}(x, t)$ and $p_{h}(x, t)$, i.e., if $n_{h}(x, t)=\sum_{j=1}^{m} n_{j}(t) \varphi_{j}(x)$ instead of (11), the equations that define the coefficients $n_{j}(t)$ and $p_{j}(t)$ have the same form as (9) and (10), but $f(z)=1-\frac{z}{2}$. Note that only when $z_{j}=0$ one obtains the same approximation to $n(x, t)$ and $p(x, t), x \in\left(x_{j-1}, x_{j}\right]$.

For the proof of convergence of the discretization scheme presented here we refer to [15].

\subsubsection{Resulting Initial Value Problem}

Let us denote by $\Psi(t), N(t)$ and $P(t)$ the unknowns of the discretized problem, i.e., $\Psi(t)=\left(\psi_{2}(t), \ldots, \psi_{m-1}(t)\right)^{T}, N(t)=\left(n_{2}(t), \ldots, n_{m-1}(t)\right)^{T}$ and $P(t)=$ $\left(p_{2}(t), \ldots, p_{m-1}(t)\right)^{T}$ for $t \in\left[t_{a}, t_{b}\right]$. The resulting initial value problem for $\Psi, N$ and $P$ can be written as an index- 1 semi-explicit DAE

$$
\begin{array}{r}
T \Psi-\left(\frac{h}{\lambda}\right)^{2}(C-N+P)-\Psi_{0}(e, t)=0, \\
\frac{\mathrm{d}}{\mathrm{d} t} N-\frac{1}{h^{2}} g_{1}(e, \Psi, N, t)+R(N, P)=0, \\
\frac{\mathrm{d}}{\mathrm{d} t} P+\frac{1}{h^{2}} g_{2}(e, \Psi, P, t)+R(N, P)=0, \\
\Psi\left(t_{a}\right)=\Psi_{a}, \quad N\left(t_{a}\right)=N_{a}, \quad P\left(t_{a}\right)=P_{a}
\end{array}
$$

where $\Psi_{0}(e, t)$ has the components ${ }^{4} \Psi_{0}(e, t)=\left(\psi_{1}(t) 0 \ldots 0 \psi_{m}(t)\right)^{T}$ and $T \in \mathbb{R}^{(m-2) \times(m-2)}$ is the tridiagonal matrix with elements

$$
T(i, i)=2, \quad T(i+1, i)=T(i, i+1)=-1 \quad i=1,2, \ldots, m-2 .
$$

The vectors $C$ and $R$ have components $C\left(x_{i+1}\right)$ and $R(N(i), P(i))$ for $i=$ $1,2, \ldots, m-2$. The functions $g_{1}$ and $g_{2}$ are vector-valued functions easily identifiable from the discretized equations. Since their expressions depend on the node potentials of the circuit, we have written them as functions of $e$ too. The vectors $N_{a}$ and $P_{a}$ represent the initial values for $N(t)$ and $P(t)$, $N_{a}=\left(n_{a}\left(x_{2}\right), \ldots, n_{a}\left(x_{m-1}\right)\right)^{T}, P_{a}=\left(p_{a}\left(x_{2}\right), \ldots, p_{a}\left(x_{m-1}\right)\right)^{T}$. If the initial

$\overline{{ }^{4} \psi_{1}(t)}=\psi(0, t)$ and $\psi_{m}(t)=\psi(l, t)$. They depend on the node potentials of the circuit. 
value for $\Psi(t)$ is such that

$$
\Psi_{a}=T^{-1}\left(\frac{h}{\lambda}\right)^{2}\left(C-N_{a}+P_{a}\right)+T^{-1} \Psi_{0}\left(e_{a}, t_{a}\right)
$$

then (12) has a locally unique solution and is equivalent to the ODE

$$
\begin{array}{r}
\frac{\mathrm{d}}{\mathrm{d} t} \Psi+T^{-1} \frac{1}{\lambda^{2}}\left(g_{1}(e, \Psi, N, t)+g_{2}(e, \Psi, P, t)\right)-T^{-1} \frac{\mathrm{d}}{\mathrm{d} t} \Psi_{0}(e, t)=0, \\
\frac{\mathrm{d}}{\mathrm{d} t} N-\frac{1}{h^{2}} g_{1}(e, \Psi, N, t)+R(N, P)=0, \\
\frac{\mathrm{d}}{\mathrm{d} t} P-\frac{1}{h^{2}} g_{2}(e, \Psi, P, t)+R(N, P)=0 .
\end{array}
$$

This ODE is obtained when the model consisting of the continuity equations and the energy conservation equation is discretized using a finite element method as described above.

\section{Coupling of the Network and Space-Discretized Drift-Diffusion Equations}

In [18], the partial differential algebraic equation that results from the coupling between the circuit equations and drift-diffusion equations for the semiconductor devices was studied as abstract differential algebraic system [11]. There it was proved that the coupled system has an index not greater than two if the assumptions in section 2 are satisfied. More precisely, it has index 2 if and only if the circuit contains LI-cut sets or CVS-loops (loops of capacitors, voltage sources and semiconductor devices) with at least one voltage source or one semiconductor device.

In this work we study the coupling between the circuit equations and discretized drift-diffusion equations for the semiconductor devices in the circuit and prove that this system has the same index under the same conditions on the circuit as the system considered in [18].

Suppose we want to couple $n_{S}$ semiconductor devices, described by discretized drift-diffusion models, to an electrical circuit. The vector $j_{S}=\left(j_{01}, \ldots, j_{0 n_{S}}\right)^{T}$ represents the current through the semiconductors. The incidence of these currents in the circuit may be described by $A_{S} j_{S}$ where $A_{S} \in \mathbb{R}^{n_{N} \times n_{S}}$ is such 
that

$$
A_{S}(i, k)=\left\{\begin{aligned}
-1, & \text { if the reference terminal of the semiconductor } k \\
& \text { is connected to node } i, \\
1, & \text { if the other terminal of the semiconductor } k \\
& \text { is connected to node } i, \\
0, & \text { else. }
\end{aligned}\right.
$$

If $\delta_{i, n_{S}}$ represents the $i$-th unitary vector of dimension $n_{S}$ and the $k$-th semiconductor device is connected to nodes $i_{k}$ and $j_{k}$ of the circuit with the reference terminal connected to node $j_{k}$, the $k$-th column of $A_{S}$ is equal to the difference between the $i$-th and the $j$-th unitary vectors, i.e., $A_{S} \delta_{k, n_{S}}=\delta_{i_{k}, n_{N}}-\delta_{j_{k}, n_{N}}$. The biases applied to the semiconductor terminals can also be described in terms of $A_{S}$

$$
\left(\begin{array}{c}
\omega_{0_{k}}(e, t) \\
\omega_{l_{k}}(e, t)
\end{array}\right)=\left(\begin{array}{c}
e_{i_{k}}(t) \\
e_{j_{k}}(t)
\end{array}\right)=\left(\begin{array}{c}
\delta_{i_{k}, n_{N}}^{T} e \\
\delta_{j_{k}, n_{N}}^{T} e
\end{array}\right)=\left(\begin{array}{c}
\delta_{i_{k}, n_{N}}^{T} e \\
\delta_{i_{k}, n_{N}}^{T} e-\delta_{k, n_{S}}^{T} A_{S}^{T} e
\end{array}\right) .
$$

Since the $t$ variable in the semiconductor equations was scaled, the circuit equations must also be scaled before coupling the discretized drift-diffusion equations to them. With

$$
\begin{aligned}
& \hat{t}=\frac{1}{t_{s}} t, \hat{e}(\hat{t})=e(t), \hat{j}_{L}(\hat{t})=j_{L}(t), \hat{j}_{V}(\hat{t})=j_{V}(t), \\
& \hat{i}_{S}(\hat{t})=i_{S}(t), \hat{v}_{S}(\hat{t})=v_{S}(t), \\
& \hat{q}\left(A_{C}^{T} \hat{e}, \hat{t}\right)=\frac{1}{t_{s}} q\left(A_{C}^{T} e, t\right), \hat{\phi}\left(\hat{j}_{L}, \hat{t}\right)=\frac{1}{t_{s}} \phi\left(j_{L}, t\right), \hat{g}\left(A_{R}^{T} \hat{e}, \hat{t}\right)=g\left(A_{R}^{T} e, t\right),
\end{aligned}
$$

the scaled circuit equations have the same form as (1)-(3).

The system that describes the behavior of the circuit containing $n_{S}$ semiconductor devices is formed by the scaled modified nodal analysis equations ${ }^{5}$, where the first one changes to [19]

$$
A_{C} \frac{\mathrm{d}}{\mathrm{d} t} q_{C}\left(A_{C}^{T} e, t\right)+A_{R} g\left(A_{R}^{T} e, t\right)+A_{L} j_{L}+A_{V} j_{V}+A_{I} i_{S}(t)+A_{S} j_{S}=0
$$

in order to include the incidence of the semiconductor devices currents into the circuit and the discretized drift-diffusion models of the $n_{S}$ semiconductor devices we want to couple to the circuit.

5 The scaled variables have been renamed as the original ones. 


\subsection{Index of the Coupled System}

Let us connect one semiconductor device to the circuit, suppose it is located between the nodes $i$ and $j$ with its reference terminal connected to node $j$. In this case, the DAE that results from the coupling of the circuit equations with the discretized semiconductor equations reads

$$
\begin{aligned}
A_{C} \frac{\mathrm{d}}{\mathrm{d} t} q_{C}\left(A_{C}^{T} e, t\right)+A_{R} g\left(A_{R}^{T} e, t\right)+A_{L} j_{L}+A_{V} j_{V}+A_{I} i_{S}(t) & +A_{S} j_{S}=0, \\
\frac{\mathrm{d}}{\mathrm{d} t} \phi\left(j_{L}, t\right)-A_{L}^{T} e & =0, \\
A_{V}^{T} e-v_{S}(t) & =0, \\
j_{S}^{d}-\beta\left(\delta_{1, m-2}^{T} \Psi-\frac{1}{U_{T}} \psi_{b i}(0)-\frac{1}{U_{T}} e_{i}\right) & =0, \\
j_{S}(t)-j_{S}^{c}(e, \Psi, N, P, t)+\frac{\mathrm{d}}{\mathrm{d} t} j_{S}^{d} & =0, \\
T \Psi-\left(\frac{h}{\lambda}\right)^{2}(C-N+P)-\Psi_{0}(e) & =0, \\
\frac{\mathrm{d}}{\mathrm{d} t} N-\frac{1}{h^{2}} g_{1}(e, \Psi, N, t)+R(N, P) & =0, \\
\frac{\mathrm{d}}{\mathrm{d} t} P+\frac{1}{h^{2}} g_{2}(e, \Psi, P, t)+R(N, P) & =0,
\end{aligned}
$$

where the function $\Psi_{0}(e)$ is

$$
\Psi_{0}(e)=\left(\frac{1}{U_{T}}\left(\psi_{b i}(0)+e_{i}\right), 0, \ldots, 0, \frac{1}{U_{T}}\left(\psi_{b i}(l)+e_{j}\right)\right)^{T}
$$

and $A_{S}$ is a column vector, $A_{S}=\delta_{i, n_{N}}-\delta_{j, n_{N}}$. In order to study the properties of (14), we will rewrite it as a DAE of the form

$$
A \frac{\mathrm{d}}{\mathrm{d} t} d(y, t)+b(y, t)=0
$$

with unknowns $y=\left(e, j_{L}, j_{V}, j_{S}, j_{S}^{d}, \Psi, N, P\right)^{T} \in \mathbb{R}^{n_{N}+n_{L}+n_{V}+1+1+3(m-2)}$. The matrix $A$ and the vectors $d$ and $b$ are

$$
A=\left(\begin{array}{ccccc}
A_{C} & 0 & 0 & 0 & 0 \\
0 & I & 0 & 0 & 0 \\
0 & 0 & 0 & 0 & 0 \\
0 & 0 & 0 & 0 & 0 \\
0 & 0 & 1 & 0 & 0 \\
0 & 0 & 0 & 0 & 0 \\
0 & 0 & 0 & I & 0 \\
0 & 0 & 0 & 0 & I
\end{array}\right), d(y, t)=\left(\begin{array}{c}
q_{C}\left(A_{C}^{T} e, t\right) \\
\phi\left(j_{L}, t\right) \\
j_{S}^{d} \\
N \\
P
\end{array}\right)
$$




$$
b(y, t)=\left(\begin{array}{c}
A_{R} g\left(A_{R}^{T} e, t\right)+A_{L} j_{L}+A_{V} j_{V}+A_{I} i_{S}(t)+A_{S} j_{S} \\
-A_{L}^{T} e \\
A_{V}^{T} e-v_{S}(t) \\
j_{S}^{d}-\beta\left(\delta_{1, m-2}^{T} \Psi-\frac{1}{U_{T}} \psi_{b i}(0)-\frac{1}{U_{T}} e_{i}\right) \\
j_{S}-j_{S}^{c}(e, \Psi, N, P, t) \\
T \Psi-\left(\frac{h}{\lambda}\right)^{2}(C-N+P)-\Psi_{0}(e, t) \\
-\frac{1}{h^{2}} g_{1}(e, \Psi, N, t)+R(N, P) \\
\frac{1}{h^{2}} g_{2}(e, \Psi, P, t)+R(N, P)
\end{array}\right) .
$$

The null space of $A$ is given by $\operatorname{ker} A=\operatorname{ker} A_{C} \times\{0\} \times\{0\} \times\{0\} \times\{0\}$. The image space of

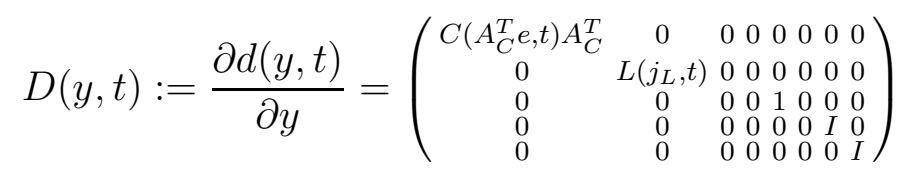

is $\operatorname{im} D(y, t)=\operatorname{im} C\left(A_{C}^{T} e, t\right) A_{C}^{T} \times \mathbb{R}^{n_{L}} \times \mathbb{R} \times \mathbb{R}^{m-2} \times \mathbb{R}^{m-2}$. The positive definiteness of $C\left(A_{C}^{T} e, t\right)$ implies that ker $A_{C} \cap \operatorname{im} C\left(A_{C}^{T} e, t\right) A_{C}^{T}=\{0\}$ and $\operatorname{dim}\left(\operatorname{ker} A_{C}\right)+\operatorname{dim}\left(\operatorname{im} C\left(A_{C}^{T} e, t\right) A_{C}^{T}\right)=n_{C}$. Then, $A$ and $D(y, t)$ satisfy

$$
\operatorname{ker} A \oplus \operatorname{im} D(y, t)=\mathbb{R}^{n_{C}+n_{L}+1+2(m-2)} \text {. }
$$

The DAE (15) with $A, d$ and $b$ as in (16) has a properly stated leading term [10] if, besides (17), the spaces $\operatorname{ker} A$ and $\operatorname{im} D(y, t)$ are independent of $y$ and have bases that are continuously differentiable in $t$ and $d(y, t) \in$ $\operatorname{im} D(y, t), \forall y, \forall t \in\left[t_{a}, t_{b}\right]$. In (16), ker $A$ is constant, but im $D(y, t)$ depends on $y$, with $\tilde{R}=A^{+} A$ it can be reformulated as ${ }^{6}$

$$
A \frac{\mathrm{d}}{\mathrm{d} t}(\tilde{R} d(y, t))+b(y, t)=0
$$

that has a properly stated leading term [14]. Due to $A \tilde{R}=A$, Backward Differentiation Formulas (BDF) and Runge-Kutta (RK) methods applied to (15) and (18) are equivalent and there is no need to compute $\tilde{R}$ in practice.

Lemma 1 If the assumptions in section 2 are satisfied and the circuit contains neither LI-cut sets nor CVS-loops with at least one voltage source or one semiconductor device, the DAE (18) has index one.

PROOF. For the index determination, we use the tractability index concept. It allows us to compute the index checking the rank of certain matrices only. Let

$$
G_{0}(y, t)=A \tilde{R} D=A D, B_{0}(y, t)=\frac{\partial b}{\partial y}(y, t), N_{0}(y, t)=\operatorname{ker} G_{0}(y, t),
$$

$\overline{6} A^{+}$denotes the Moore-Penrose pseudo inverse of $A$. 
$Q_{0}$ be a projector onto $N_{0}$ and $G_{1}=G_{0}+B_{0} Q_{0}$. If $G_{0}$ is singular with constant rank and $G_{1}$ is non-singular, the DAE has tractability index one. Note that

$$
N_{0}(y, t)=N_{0}=\left\{y \mid y_{e} \in \operatorname{ker} A_{C}^{T}, y_{L}=y_{S}^{d}=y_{N}=y_{P}=0\right\}
$$

and, if $Q_{C}$ denotes a projector onto $\operatorname{ker} A_{C}^{T}$, the matrix

$$
Q_{0}=\left(\begin{array}{cccccccc}
Q_{C} & 0 & 0 & 0 & 0 & 0 & 0 & 0 \\
0 & 0 & 0 & 0 & 0 & 0 & 0 & 0 \\
0 & 0 & I & 0 & 0 & 0 & 0 & 0 \\
0 & 0 & 0 & 1 & 0 & 0 & 0 & 0 \\
0 & 0 & 0 & 0 & 0 & 0 & 0 & 0 \\
0 & 0 & 0 & 0 & 0 & I & 0 & 0 \\
0 & 0 & 0 & 0 & 0 & 0 & 0 & 0 \\
0 & 0 & 0 & 0 & 0 & 0 & 0 & 0
\end{array}\right)
$$

is a projector onto $N_{0}$. Then

$$
G_{1}=\left(\begin{array}{cccccccc}
A_{C} C\left(A_{C}^{T} e, t\right) A_{C}^{T}+A_{R} G\left(A_{R}^{T} e, t\right) A_{R}^{T} Q_{C} & 0 & A_{V} & A_{S} & 0 & 0 & 0 & 0 \\
-A_{L}^{T} Q_{C} & L\left(j_{L}, t\right) & 0 & 0 & 0 & 0 & 0 & 0 \\
A_{V}^{T} Q_{C} & 0 & 0 & 0 & 0 & 0 & 0 & 0 \\
\frac{\beta}{U_{T}} \delta_{i, n_{N}}^{T} Q_{C} & 0 & 0 & 0 & 0 & -\beta \delta_{1, m-2}^{T} & 0 & 0 \\
-\frac{\partial j_{S}^{c}}{\partial e} Q_{C} & 0 & 0 & 1 & 1 & -\frac{\partial j_{S}^{c}}{\partial \Psi} & 0 & 0 \\
-\frac{1}{U_{T}}\left(\delta_{i, n_{N}} 0 \ldots \delta_{j, n_{N}}\right)^{T} Q_{C} & 0 & 0 & 0 & 0 & T & 0 & 0 \\
-\frac{1}{h^{2}} \frac{\partial g_{1}}{\partial e} Q_{C} & 0 & 0 & 0 & 0 & -\frac{1}{h^{2}} \frac{\partial g_{1}}{\partial \Psi} & I & 0 \\
\frac{1}{h^{2}} \frac{\partial g_{2}}{\partial e} Q_{C} & 0 & 0 & 0 & 0 & \frac{1}{h^{2}} \frac{\partial g_{2}}{\partial \Psi} & 0 & I
\end{array}\right) .
$$

The vector $y=\left(\begin{array}{lllll}y_{e} & y_{L} & y_{V} & y_{S} & y_{S}^{d} y_{\Psi} y_{N} y_{P}\end{array}\right)^{\mathrm{T}}$ belongs to $\operatorname{ker} G_{1}$ if and only if it satisfies

$$
\begin{array}{r}
y_{L}=L(\cdot)^{-1} A_{L}^{T} Q_{C} y_{e}, \quad y_{N}=\frac{1}{h^{2}}\left(\frac{\partial g_{1}}{\partial e} Q_{C} y_{e}+\frac{\partial g_{1}}{\partial \Psi} y_{\Psi}\right) \\
y_{P}=-\frac{1}{h^{2}}\left(\frac{\partial g_{2}}{\partial e} Q_{C} y_{e}+\frac{\partial g_{2}}{\partial \Psi} y_{\Psi}\right), \quad y_{S}^{d}=\frac{\partial j_{S}^{c}}{\partial e} Q_{C} y_{e}-y_{S}+\frac{\partial j_{S}^{c}}{\partial \Psi} y_{\Psi}, \\
y_{\Psi}=\frac{1}{U_{T}} T^{-1}\left(\delta_{i, n_{N}} 0 \cdots 0 \delta_{j, n_{N}}\right)^{T} Q_{C} y_{e}, \\
A_{C} C(\cdot) A_{C}^{T} y_{e}+A_{R} G(\cdot) A_{R}^{T} Q_{C} y_{e}+A_{V} y_{V}+A_{S} y_{S}=0, \\
A_{V}^{T} Q_{C} y_{e}=0, \\
\frac{1}{U_{T}} \delta_{i, n_{N}}^{T} Q_{C} y_{e}-\delta_{1, m-2}^{T} y_{\Psi}=0 .
\end{array}
$$

Inserting $y_{\Psi}$ from (19c) into (19f) yields $A_{S}^{T} Q_{C} y_{e}=0$ because $T$ satisfies $^{7}$ $T^{-1}(1,1)+T^{-1}(1, m-2)=1$.

$\overline{7}$ The matrix $T$ of size $k \in \mathbb{N}$ is a symmetric matrix of the form

$$
T_{k}=\left(\begin{array}{cc}
\alpha_{k+1}+\alpha_{k} & -\alpha_{k} \delta_{1, k-1}^{T} \\
-\alpha_{k} \delta_{1, k-1} & T_{k-1}
\end{array}\right) .
$$

This means that its inverse is also a symmetric matrix that can be written as $T_{k}^{-1}=\left(\begin{array}{cc}a & b^{T} \\ b & C\end{array}\right)$ with $b$ a $(k-1)$-dimensional vector and $C$ a $(k-1) \times(k-1)$ matrix, the scalar $a$ and the vector $b$ must then satisfy the $k$ relations $\left(\alpha_{k+1}+\alpha_{k}\right) a-$ $\alpha_{k} \delta_{1, k-1}^{T} b=1$ and $-\alpha_{k} \delta_{1, k-1} a+T_{k-1} b=0$, adding the last $k-1$ equations one 
Let $Q_{V S}$ be a projector onto $\operatorname{ker}\left(\begin{array}{c}A_{V}^{T} Q_{C} \\ A_{S}^{T} Q_{C}\end{array}\right)$, then $Q_{V S} y_{e}=y_{e}$ and

$$
Q_{V S}^{T} Q_{C}^{T} A_{V}=Q_{V S}^{T} Q_{C}^{T} A_{S}=0 .
$$

Multiplying equation (19d) by $Q_{V S}^{T} Q_{C}^{T}$ one obtains that $y_{e}$ must also satisfy $A_{R}^{T} Q_{C} y_{e}=0$ (remember that $G(\cdot)$ is positive definite). Consequently, $Q_{C} y_{e}$ belongs to ker $\left(A_{C} A_{V} A_{R} A_{S}\right)^{T}$ if we regard the previous conditions for $Q_{C} y_{e}$ and take into account that $A_{C}^{T} Q_{C} y_{e}=0$.

Since $Q_{C R V S}$ is a projector onto $\operatorname{ker}\left(A_{C} A_{R} A_{V} A_{S}\right)^{T}$, it holds that

$$
Q_{C R V S} Q_{C} y_{e}=Q_{C} y_{e}
$$

Then, equation (19d) implies that $y_{e}, y_{L}, y_{V}$ satisfy

$$
A_{C} C(\cdot) A_{C}^{T} Q_{C} y_{e}+A_{V} y_{V}+A_{S} y_{S}=0 .
$$

Multiplying this relation by $Q_{C}^{T}$ one obtains that $y_{V}, y_{S}$ fulfill

$$
Q_{C}^{T} A_{V} y_{V}+Q_{C}^{T} A_{S} y_{S}=0
$$

i.e., $\left(y_{V} y_{S}\right)^{T} \in \operatorname{ker}\left(Q_{C}^{T} A_{V} Q_{C}^{T} A_{S}\right)$.

If the circuit does not have LI-cut sets, the matrix $\left(A_{C} A_{R} A_{V} A_{S}\right)^{T}$ has full column rank and $Q_{C} y_{e}=0$. If the circuit does not contain CVS-loops with at least one voltage source or one semiconductor device, the matrix $\left(Q_{C}^{T} A_{V} Q_{C}^{T} A_{S}\right)$ has full column rank and then $\left(y_{V} y_{S}\right)^{T}=0$. Hence, condition (19d) implies $y_{e} \in \operatorname{ker} A_{C} C(\cdot) A_{C}^{T}=\operatorname{ker} A_{C}^{T}$, i.e., $y_{e}=Q_{C} y_{e}=0$. Finally, $y_{L}=y_{\Psi}=y_{N}=y_{P}=y_{S}^{d}=0$ and $G_{1}$ is a non-singular matrix.

Due to the results in [10] it can be assured that, under the assumptions of Lemma 1, the system (18) has also perturbation index one. Furthermore, if the initial value $y_{a}=\left(e_{a}, j_{L a}, j_{V a}, j_{S a}, j_{S a}^{d}, \Psi_{a}, N_{a}, P_{a}\right)^{T}$ satisfies

$$
\begin{aligned}
Q_{C}^{T}\left(A_{R} g\left(A_{R}^{T} e_{a}, t_{a}\right)+A_{L} j_{L a}+A_{V} j_{V a}+A_{I} i_{S}\left(t_{a}\right)+A_{S} j_{S a}\right) & =0, \\
A_{V}^{T} e_{a}-v_{S}\left(t_{a}\right) & =0, \\
T \Psi_{a}-\left(\frac{h}{\lambda}\right)^{2}\left(C-N_{a}+P_{a}\right)-\Psi_{0}\left(e_{a}, t_{a}\right) & =0, \\
j_{S a}^{d}-\beta\left(\delta_{1, m-2}^{T} \Psi_{a}-\frac{1}{U_{T}}\left(\psi_{b i}(0)+\delta_{i, n_{N}}^{T} e_{a}\right)\right) & =0
\end{aligned}
$$

the DAE (18) is uniquely solvable. In addition, BDF and RK methods applied to its numerical solution, are convergent.

$\overline{\text { obtains }}$ that $\alpha_{k} b_{1}+\alpha_{1} b_{k-1}=\alpha_{k} a$ that together with the first relation implies that $\alpha_{k+1} a+\alpha_{1} b_{k-1}=1$. 
Suppose the circuit contains LI-cut sets or CVS-loops with at least one voltage source or one semiconductor device. Let $Q_{C-V S}$ denote a projector onto $\operatorname{ker} Q_{C}^{T}\left(A_{V} A_{S}\right)$. Then, the vector $y$ belongs to $\operatorname{ker} G_{1}$ if conditions (19a)-(19c) are satisfied and

$A_{C} C(\cdot) A_{C}^{T} P_{C} y_{e}+A_{V} y_{V}+A_{S} y_{S}=0, Q_{C} y_{e}=Q_{C R V S} Q_{C} y_{e},\left(\begin{array}{l}y_{V} \\ y_{S}\end{array}\right)=Q_{C-V S}\left(\begin{array}{l}y_{V} \\ y_{S}\end{array}\right)$.

Since im $Q_{C R V S} \subseteq \operatorname{im} Q_{C}$, the projector $Q_{C R V S}$ may be constructed such that $\operatorname{ker} Q_{C} \subseteq \operatorname{ker} Q_{C R V S}$. The vector $y \in \operatorname{ker} G_{1}$ may then be described by conditions (19a)-(19c) and

$$
\begin{aligned}
& P_{C} y_{e}=-H_{C}(\cdot)^{-1}\left(A_{V} A_{S}\right) Q_{C-V S}\left(y_{V} y_{S}\right)^{T}, \\
& Q_{C} y_{e}=Q_{C R V S}\left(y_{e}+Q_{C} y_{e}-y_{e}\right)=Q_{C R V S} y_{e},\left(\begin{array}{l}
y_{V} \\
y_{S}
\end{array}\right)=Q_{C-V S}\left(\begin{array}{l}
y_{V} \\
y_{S}
\end{array}\right),
\end{aligned}
$$

where the matrix $H_{C}(\cdot)=A_{C} C(\cdot) A_{C}^{T}+Q_{C}^{T} Q_{C}$ is positive definite. Because of $P_{C}^{T} H_{C}(\cdot)=H_{C}(\cdot) P_{C}$, we get

$$
Q_{C} H_{C}(\cdot)^{-1}\left(A_{V} A_{S}\right) Q_{C-V S}=H_{C}(\cdot)^{-1}\left(Q_{C}^{T} A_{V} Q_{C}^{T} A_{S}\right) Q_{C-V S}=0
$$

and $P_{C} H_{C}(\cdot)^{-1}\left(A_{V} A_{S}\right) Q_{C-V S}=H_{C}(\cdot)^{-1}\left(A_{V} A_{S}\right) Q_{C-V S}$.

If we denote $C_{L}=L(\cdot)^{-1} A_{L}^{T}, C_{N}=\frac{1}{h^{2}}\left(\frac{\partial g_{1}}{\partial e}+\frac{\partial g_{1}}{\partial \Psi} C_{\Psi}\right), C_{P}=-\frac{1}{h^{2}}\left(\frac{\partial g_{2}}{\partial e}+\frac{\partial g_{2}}{\partial \Psi} C_{\Psi}\right)$, $C_{j_{S}^{d}}=\left(\frac{\partial j_{S}^{c}}{\partial e}+\frac{\partial j_{S}^{c}}{\partial \Psi} C_{\Psi}\right)$ and $C_{\Psi}=\frac{1}{U_{T}} T^{-1}\left(\delta_{i, n_{N}} 0 \cdots \cdots \delta_{j, n_{N}}\right)^{T}$, a projector $Q_{1}$ onto ker $G_{1}$ can be written as

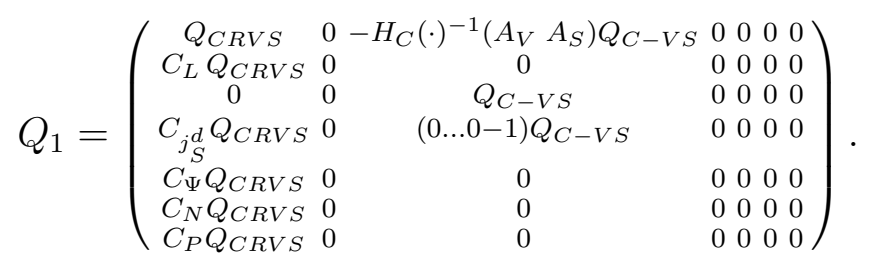

Lemma 2 If the assumptions in section 2 are satisfied, the circuit contains LI-cut sets or CVS-loops with at least one voltage source or one semiconductor device and $N$ and $P$ are always greater than zero, the DAE (18) has index 2.

PROOF. Again, we use the tractability index concept for the index determination. The DAE has tractability index two if the matrix $G_{1}$ is singular and has constant rank and $G_{2}=G_{1}+B_{0} P_{0} Q_{1}$ is non-singular. It can be proved that if $N$ and $P$ are always greater than zero the matrix $G_{1}$ has constant rank $^{8}$. It remains to show that $G_{2}$ is non-singular.

8 By looking at the structure of $Q_{1}$ one sees that it has constant rank if the products $C_{N} Q_{C R V S}, C_{P} Q_{C R V S}$ and $C_{j_{S}^{d}} Q_{C R V S}$ have constant rank. Using that $\operatorname{dim}(\operatorname{im} A B)=\operatorname{dim}(\operatorname{im} B)-\operatorname{dim}(\operatorname{im} B \cap \operatorname{ker} A)$ the desired result is obtained. 
In $B_{0} P_{0} Q_{1}$, only the first, third and fourth columns are different to zero, they are

$$
\left(\begin{array}{c}
A_{L} C_{L} Q_{C R V S} \\
0 \\
0 \\
C_{j_{S}^{d}} Q_{C R V S} \\
-\left(\frac{\partial j_{S}^{c}}{\partial N} C_{N}+\frac{\partial j_{S}^{c}}{\partial P} C_{P}\right) Q_{C R V S} \\
\left(\frac{h}{\lambda}\right)^{2}\left(C_{N}-C_{P}\right) Q_{C R V S} \\
\left(\left(-\frac{1}{h^{2}} \frac{\partial g_{1}}{\partial N}+\frac{\partial R}{\partial N}\right) C_{N}+\frac{\partial R}{\partial P} C_{P}\right) Q_{C R V S} \\
\left(\left(\frac{1}{h^{2}} \frac{\partial g_{2}}{\partial P}+\frac{\partial R}{\partial P}\right) C_{P}+\frac{\partial R}{\partial N} C_{N}\right) Q_{C R V S}
\end{array}\right),\left(\begin{array}{c}
-A_{R} G(\cdot) A_{R}^{T} H_{C}(\cdot)^{-1}\left(A_{V} A_{S}\right) Q_{C-V S} \\
A_{L}^{T} H_{C}(\cdot)^{-1}\left(A_{V} A_{S}\right) Q_{C-V S} \\
-A_{V}^{T} H_{C}(\cdot)^{-1}\left(A_{V} A_{S}\right) Q_{C-V S} \\
-\frac{\beta}{U_{T}} \delta_{i, n_{C}}^{T} H_{C}(\cdot)^{-1}\left(A_{V} A_{S}\right) Q_{C-V S}+(0 \cdots 0-1) Q_{C-V S} \\
\frac{\partial j_{S}^{c}}{\partial e} H_{C}(\cdot)^{-1}\left(A_{V} A_{S}\right) Q_{C-V S} \\
\frac{1}{U_{T}}\left(\delta_{i, n_{N}} \cdots \cdots \delta_{j, n_{N}}\right)^{T} H_{C}(\cdot)^{-1}\left(A_{V} A_{S}\right) Q_{C-V S} \\
\frac{1}{h^{2}} \frac{\partial g_{1}}{\partial e} H_{C}(\cdot)^{-1}\left(A_{V} A_{S}\right) Q_{C-V S} \\
-\frac{1}{h^{2}} \frac{\partial g_{2}}{\partial e} H_{C}(\cdot)^{-1}\left(A_{V} A_{S}\right) Q_{C-V S}
\end{array}\right) .
$$

Suppose the vector $y=\left(y_{e} y_{L} y_{V} y_{S} y_{S^{d}} y_{\Psi} y_{N} y_{P}\right)^{T}$ belongs to the null space of $G_{2}$. Multiplying the first equation of $G_{2} y=0$ by $Q_{C R V S}^{T}$ one obtains $Q_{C R V S}^{T} A_{L} L(\cdot)^{-1} A_{L}^{T} Q_{C R V S} y_{e}=0$. Since $L(\cdot)$ is positive definite, this is equivalent to $A_{L}^{T} Q_{C R V S} y_{e}=0$. Due to the assumption that the circuit does not contain cut sets of current sources only, the matrix $\left(A_{C} A_{L} A_{R} A_{V} A_{S}\right)^{T}$ has full column rank and, consequently, $A_{L}^{T} Q_{C R V S} y_{e}=0 \Leftrightarrow Q_{C R V S} y_{e}=0$.

Inserting $y_{\Psi}$ from the sixth equation of $G_{2} y=0$ into the fourth and taking into account that the matrix $T$ satisfies that $T^{-1}(1,1)+T^{-1}(1, m-2)=1$, the components $y_{e}, y_{V}$ and $y_{S}$ of $y$ must satisfy

$$
\delta A_{S}^{T}\left(Q_{C} y_{e}-H_{C}(\cdot)^{-1}\left(A_{V} A_{S}\right) Q_{C-V S}\left(\begin{array}{l}
y_{V} \\
y_{S}
\end{array}\right)\right)=\left(0 \cdots 0 \frac{1}{\beta}\right) Q_{C-V S}\left(\begin{array}{l}
y_{V} \\
y_{S}
\end{array}\right)
$$

where $\delta=\frac{T^{-1}(1, m-2)}{U_{T}}$. The last condition and the third equation of $G_{2} y=0$ may be written as

$$
\left(\begin{array}{c}
A_{V}^{T} \\
A_{S}^{T}
\end{array}\right) Q_{C} y_{e}-\left(\begin{array}{c}
A_{V}^{T} \\
A_{S}^{T}
\end{array}\right) H_{C}(\cdot)^{-1}\left(A_{V} A_{S}\right) Q_{C-V S}\left(\begin{array}{c}
y_{V} \\
y_{S}
\end{array}\right)=\left(\begin{array}{cccc}
0 & \cdots & 0 & 0 \\
\vdots & \vdots & \vdots & \vdots \\
0 & \cdots & 0 & \frac{1}{\beta \delta}
\end{array}\right) Q_{C-V S}\left(\begin{array}{c}
y_{V} \\
y_{S}
\end{array}\right) .
$$

Multiplying it by $Q_{C-V S}^{T}$, we get

$$
Q_{C-V S}^{T}\left(\left(A_{V} A_{S}\right)^{T} H_{C}(\cdot)^{-1}\left(A_{V} A_{S}\right)+\left(\begin{array}{cccc}
0 & \cdots & 0 & 0 \\
\vdots & \vdots & \vdots & \vdots \\
0 & \cdots & 0 & 0 \\
0 & \cdots & 0 & \frac{1}{\beta \delta}
\end{array}\right)\right) Q_{C-V S}\left(\begin{array}{l}
y_{V} \\
y_{S}
\end{array}\right)=0 .
$$

Because the matrices in this sum are positive definite, it is zero if and only if

$$
\left(A_{V} A_{S}\right) Q_{C-V S}\left(\begin{array}{l}
y_{V} \\
y_{S}
\end{array}\right)=0 \quad \text { and } \quad Q_{C-V S}\left(\begin{array}{c}
y_{V} \\
y_{S}
\end{array}\right) \in \operatorname{ker}\left(\begin{array}{cccc}
0 & \cdots & 0 & 0 \\
\vdots & \vdots & \vdots & \vdots \\
0 & \cdots & 0 & 0 \\
0 & \cdots & 0 & \frac{1}{\beta \delta}
\end{array}\right) .
$$

If $\left(v_{1} v_{2}\right)^{T}=Q_{C-V S}\left(\begin{array}{l}y_{V} \\ y_{S}\end{array}\right)$, the above conditions imply $v_{2}=0$ and $A_{V} v_{1}+$ $A_{S} v_{2}=0$. Since $A_{V}$ has full column rank we find $v_{1}=0$. Regarding. $Q_{C R V S} y_{e}=$ $Q_{C-V S}\left(\begin{array}{l}y_{V} \\ y_{S}\end{array}\right)=0$, it holds that $B_{0} P_{0} Q_{1} y=0$. Thus, $y$ belongs to $\operatorname{ker} G_{2}$ if 
and only if it belongs to $\operatorname{ker} G_{1}$, i.e., if $y=Q_{1} y$. This implies $Q_{C R V S} y_{e}=$ $Q_{C-V S}\left(\begin{array}{l}y_{V} \\ y_{S}\end{array}\right)=0$ and $y=Q_{1} y=0$.

Due to the results in [13], it can be assured that, under the assumptions of Lemma 2, the DAE (18) has also perturbation index two.

Following the steps in lemmata 1 and 2, it is easy to prove that the results remain the same for a nonuniform spatial mesh and circuits containing more than one semiconductor device. Furthermore, the index results do not change when standard finite elements are used to approximate the functions $n(x, t)$ and $p(x, t)$.

Lemma 3 The DAE that originates from the coupling of the ODE (13) to the circuit equations can also be written as a DAE with properly stated leading term and has the same index as the DAE previously analyzed.

PROOF. This DAE can be written as a DAE of the form $\bar{A} \frac{\mathrm{d}}{\mathrm{d} t} \bar{d}(y, t)+\bar{b}(y, t)=$ 0 with

$$
\begin{aligned}
& \bar{A}=\left(\begin{array}{cccccc}
A_{C} & 0 & 0 & 0 & 0 & 0 \\
0 & I & 0 & 0 & 0 & 0 \\
0 & 0 & 0 & 0 & 0 & 0 \\
0 & 0 & 0 & 0 & 0 & 0 \\
0 & 0 & 1 & 0 & 0 \\
0 & 0 & 0 & 0 & 0 & 0 \\
0 & 0 & 0 & 0 & 0 & I \\
0 & 0 & 0 & 0 & 0 & I
\end{array}\right), \bar{d}(y, t)=\left(\begin{array}{c}
q_{C}\left(A_{C}^{T} e, t\right) \\
\phi\left(j_{L}, t\right) \\
j_{S}^{d} \\
T \Psi \\
N \\
P \\
P
\end{array}\right) \\
& \bar{b}(y, t)=\left(\begin{array}{c}
A_{R} g\left(A_{R}^{T} e, t\right)+A_{L} j_{L}+A_{V} j_{V}+A_{I} i_{S}(t)+A_{S} j_{S} \\
-A_{L}^{T} e \\
A_{V}^{T} e-v_{S}(t) \\
j_{S}^{d}-\beta\left(\delta_{1, m-2}^{T} \Psi-\frac{1}{U_{T}}\left(\psi_{b i}(0)-e_{i}\right)\right) \\
j_{S}-j_{S}^{c}(e, \Psi, N, P, t) \\
\frac{1}{\lambda^{2}}\left(g_{1}(e, \Psi, N, t)+g_{2}(e, \Psi, N, t)\right) \\
-\frac{1}{h^{2}} g_{1}(e, \Psi, N, t)+R(N, P) \\
\frac{1}{h^{2}} g_{2}(e, \Psi, P, t)+R(N, P)
\end{array}\right) .
\end{aligned}
$$

In this case, $\operatorname{ker} \bar{A} \oplus \operatorname{im} \bar{D}(y, t)=\mathbb{R}^{n_{C}+n_{L}+3(m-2)+1}$. The null space $\bar{N}_{0}$ of $\bar{G}_{0}$ is

$$
\bar{N}_{0}=N_{0} \cap\left\{y \mid y_{\Psi}=\frac{1}{U_{T}} T^{-1}\left(\delta_{i, n_{N}} 0 \ldots 0 \delta_{j, n_{N}}\right)^{T} y_{e}\right\}
$$

and a projector $\bar{Q}_{0}$ onto $\bar{N}_{0}$ can then be written as

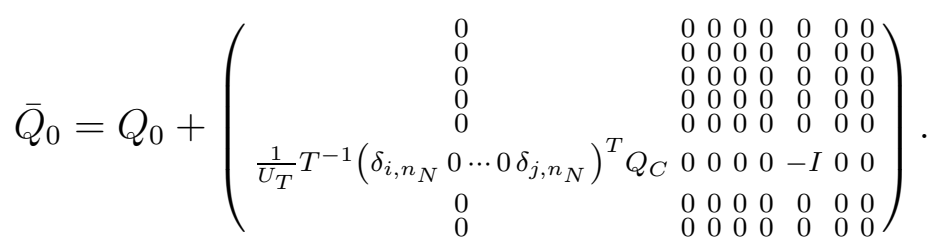


Following the steps in the proof of lemma 1 it can be proved that the DAE has index one if the circuit contains neither LI-cut sets nor CVS-loops with at least one voltage source or one semiconductor device. A projector $\bar{Q}_{1}$ onto the null space of $\bar{G}_{1}$ is now

$$
\bar{Q}_{1}=\left(\begin{array}{ccccccc}
Q_{C R V S} & 0 & -H_{C}(\cdot)^{-1}\left(A_{V} A_{S}\right) Q_{C-V S} & 0 & 0 & 0 & 0 \\
C_{L} Q_{C R V S} & 0 & 0 & 0 & 0 & 0 & 0 \\
0 & 0 & Q_{C-V S} & 0 & 0 & 0 & 0 \\
C_{j_{S}} Q_{C R V S} & 0 & (0 \ldots 0-1) Q_{C-V S} & 0 & 0 & 0 & 0 \\
\bar{C}_{\Psi} Q_{C R V S} & 0 & -C_{\Psi} H_{C}(\cdot)^{-1}\left(A_{V} A_{S}\right) Q_{C-V S} & 0 & 0 & 0 & 0 \\
C_{N} Q_{C R V S} & 0 & 0 & 0 & 0 & 0 & 0 \\
C_{P} Q_{C R V S} & 0 & 0 & 0 & 0 & 0 & 0
\end{array}\right)
$$

with $\bar{C}_{\Psi}=C_{\Psi}-\left(\frac{h}{\lambda}\right)^{2} T^{-1}\left(C_{N}-C_{P}\right)$.

In a very similar way as in lemma 2 it can be proved that also in this case the DAE has tractability index two if the circuit contains LI-cut sets or CVS-loops with at least one voltage source or one semiconductor device.

\section{Summary}

Electrical circuits containing semiconductor devices can be modeled as a coupled system of differential algebraic and partial differential equations. An approximate solution of such a system can be obtained, as proposed here, by discretizing the partial differential equations in space and solving numerically the resulting DAE. In order to gain information about how to choose consistent initial values, what type of numerical methods may be used for the solution of this DAE, etc., it is important to determine its index.

In the lemmata 1 and 2 , the special case of an electrical circuit containing only one semiconductor device modeled by one-dimensional drift-diffusion equations was studied. We proved that the resulting DAE has always index smaller or equal to two. It can be determined by topological conditions on the circuit only. These results can easily be generalized to circuits with more semiconductor devices. We expect that if drift-diffusion equations in two or three spatial dimensions are used to model the semiconductor devices in the circuit the index conditions will be very similar.

Because for the numerical solution of the drift-diffusion equations it is sometimes recommended to replace the Poisson equation by the energy conservation equation we also studied the DAE resulting from the coupling of the circuit equations and the ODE (13). In lemma 3 it was proved that the results about the tractability index are also valid for this DAE.

For the numerical solution of the coupled system we have made some experiments with a coupling between the device simulator TeSCA [9] developed at 
Weierstrass Institute in Berlin and DASSL [2]. It is our intention now to implement a software for the solution of the whole DAE that is not based on the coupling of the two simulators. Comparisons between both approaches will be the subject of a future work.

\section{Acknowledgements}

The authors wish to thank R. Lamour, D. Díaz, A. Jüngel and R. Nürnberg for very fruitful discussions.

\section{References}

[1] G. Ali, Bartel A., Günther M., Tischendorf C., Elliptic Partial Differential Algebraic Multiphysics Models in Electrical Network Design, Technical Report, Institut of Scientific Computing and Mathematical Modelling, University of Karlsruhe, 5, 2002.

[2] Brenan K. E., Campbell S. L., Petzold L. R., The numerical solution of Initial Value Problems in Ordinary Differential Algebraic Equations, North Holland Publishing Co., 1989.

[3] Estévez Schwarz D., Tischendorf C., Structural analysis of electrical circuits and consequences for MNA, Int. J. Circuit Theory and Applications, 28(2000), $131-162$.

[4] Fosséprez M., Non-linear Circuits: Qualitative Analysis of Non-linear, Nonreciprocal Circuits, John Wiley \& Sons, Chichester, 1992.

[5] Gajewski H., On Existence, uniqueness and asymptotic behaviour of solutions of the basic equations for carrier transport in semiconductors, Zeitschrift für Angewandte Mathematik und Mechanik, 65(1985), 101-108.

[6] Gajewski H., Gröger K., Semiconductor equations for variable mobilities based on Boltzmann statistics or Fermi-Dirac statistics, Math. Nachr., 140(1989), $7-36$.

[7] Gajewski H., Analysis und Numerik von Ladungstransport in Halbleitern, Technical Report, Weierstrass Institut für Andgewandte Analysis und Stochastik, 6, 1993.

[8] Gajewski H., On the uniqueness of solutions to the Drift-Diffusion model of semiconductors devices, Mathematical Models and Methods in Applied Sciences, 4(1994), 121-133. 
[9] Gajewski H., Heinemann B., Langmach H., Nürnberg R., Kaiser H.-Chr., Bandelow U., WIAS-TeSCA. Two dimensional Semi-Conductor Analysis Package, Manual, Weierstrass Institut für Angewandte Analysis und Stochastik, 1999.

[10] Higueras I., März R., Differential algebraic equations with properly stated leading terms, to appear in Comp. Math. Appl., 2003.

[11] Lamour R., März R., Tischendorf C., PDAEs and Further Mixed Systems as Abstract Differential Algebraic Systems, Technical Report, Institute of Mathematics, Humboldt University of Berlin, 11, 2001.

[12] Markovich P.A., The stationary semiconductor device equations, SpringerVerlag, 1986.

[13] März R., Nonlinear differential-algebraic equations with properly formulated leading term, Technical Report, Humboldt University of Berlin, 3, 2001.

[14] März R., Differential algebraic systems with properly stated leading term and MNA equations, in: K. Antreich, R. Burlisch, A. Gilg and P. Rentrop (Eds), Modeling, Simulation and Optimization of Integrated Circuits, International Series of Numerical Mathematics, Vol. 146, 2003, pp. 135-151.

[15] Mock M. S., Analysis of Mathematical Models of Semiconductor Devices, Boole Press, 1983.

[16] Selberherr S., Analysis and simulation of semiconductor devices, SpringerVerlag, 1984.

[17] Tischendorf C., Topological index calculation of DAEs in circuit simulation, Surv. Math. Ind., 8(1999), 187-199.

[18] Tischendorf C., Modeling circuit systems coupled with distributed semiconductor equations, in: K. Antreich, R. Burlisch, A. Gilg and P. Rentrop (Eds), Modeling, Simulation and Optimization of Integrated Circuits, International Series of Numerical Mathematics, Vol. 146, 2003, pp. 229-247.

[19] Tischendorf C., Coupled Systems of Differential Algebraic and Partial Differential Equations in Circuit and Device Simulation, Habilitation Thesis, Humboldt University of Berlin, 2003. 